\title{
NCYM is upregulated by lncUSMycN and modulates N-Myc expression
}

\author{
PEI Y. LIU ${ }^{1}$, BERNARD ATMADIBRATA ${ }^{1}$, SUJANNA MONDAL $^{1}$, ANDREW E. TEE ${ }^{1}$ and TAO LIU ${ }^{1,2}$ \\ ${ }^{1}$ Children's Cancer Institute Australia for Medical Research, Randwick, Sydney, NSW 2031; \\ ${ }^{2}$ Centre for Childhood Cancer Research, UNSW Australia, Sydney, NSW 2052, Australia
}

Received August 8, 2016; Accepted September 27, 2016

DOI: 10.3892/ijo.2016.3730

\begin{abstract}
Neuroblastoma is the most common solid tumor in early childhood. Patients with neuroblastoma due to the amplification of a 130-kb genomic DNA region containing the $M Y C N, M Y C N$ antisense NCYM and lncUSMycN genes show poor prognosis. BET bromodomain inhibitors show anticancer efficacy against neuroblastoma partly by reducing $M Y C N$ gene transcription and N-Myc mRNA and protein expression. We have previously shown that the long nocoding RNA lncUSMycN upregulates N-Myc mRNA expression by binding to the RNA-binding protein NonO. In this study, we found that IncUSMycN upregulated NCYM expression, and knockingdown lncUSMycN reduced histone H3 lysine 4 trimethylation, a marker for active gene transcription, at the NCYM gene promoter. NCYM upregulated N-Myc mRNA expression, NCYM RNA formed a complex with NonO protein, and knocking down NCYM expression reduced neuroblastoma cell proliferation. Importantly, treatment with BET bromodomain inhibitors reduced NCYM expression. In human neuroblastoma patients, high levels of NCYM expression in tumor tissues correlated with high levels of N-Myc, NonO and lncUSMycN expression as well as poor patient prognosis. Taken together, our findings suggest that lncUSMycN upregulates NCYM expression by activating its gene transcription, and that NCYM RNA upregulates N-Myc mRNA expression by binding to NonO. Our findings also provide further evidence for the application of BET bromodomain inhibitors for the therapy of neuroblastoma characterized by MYCN/ NCYM gene locus amplification.
\end{abstract}

\section{Introduction}

Neuroblastoma is the most common solid tumor in children less than 5 years old, and is derived from primordial neural

Correspondence to: Dr Tao Liu, Children's Cancer Institute Australia for Medical Research, Randwick, Sydney, NSW 2031, Australia

E-mail: tliu@ccia.unsw.edu.au

Key words: neuroblastoma, NCYM, N-Myc, lncUSMycN crest cells that eventually reside in the sympathetic nervous system $(1,2)$. Amplification of a $130 \mathrm{~kb}$ core genomic DNA region containing the $M Y C N$ oncogene, the $M Y C N$ antisense $N C Y M$ gene and the long non-coding RNA $\operatorname{lnc} U S M y c N$ gene, occurs in more than a quarter of human neuroblastoma tissues (3). More than $70 \%$ of patients with neuroblastoma associated with the amplification of the $130 \mathrm{~kb}$ core genomic DNA region die of the disease in spite of current intensive multimodal therapies $(4,5)$.

The $M Y C N$ oncogene encodes the transcription factor $\mathrm{N}-\mathrm{Myc}$ which plays important roles in cell proliferation and differentiation during embryonic development (6), and induces neuroblastoma initiation and progression by regulating target gene expression (7).

Long non-coding RNAs are transcripts longer than 200 nucleotides without protein-coding potential, and can be divided into five different classes according to their gene position relevant to neighbouring protein-coding genes: sense, antisense, bidirectional, intronic and intergenic $(8,9)$. Long non-coding RNAs have recently emerged as important regulators of gene transcription, malignant transformation, tumor initiation, progression and metastasis $(10,11)$. For example, lincRNA-p21 is activated by the tumor suppressor p53, represses the transcription of the genes that interfere with apoptosis, and plays a critical role in p53-mediated apoptosis (12). The long non-coding RNA NORAD is induced after DNA damage, and maintains genomic stability by sequestering PUMILIO proteins (13). We have recently shown that the long non-coding RNA lncUSMycN increases N-Myc mRNA expression by interacting with the RNA-binding protein NonO (14).

While N-Myc has been extensively studied, the function of the antisense NCYM gene did not attract research until recently. NCYM has been shown to stabilize N-Myc protein by inhibiting the activity of GSK $3 \beta$, a kinase that promotes $\mathrm{N}$-Myc protein degradation (15), and to promote calpainmediated Myc-nick production in human neuroblastoma cells (16). In addition, NCYM plays an essential role in neuroblastoma cell metastasis (15). In this study, we examined how NCYM gene expression was regulated, whether NCYM regulated the expression of its neighbouring protein-coding gene $M Y C N$, and whether a high level of NCYM expression in human neuroblastoma tissues was a marker for poor patient survival. 


\section{Materials and methods}

Cell culture. Neuroblastoma BE(2)-C cells were cultured in Dulbecco's modified Eagle's medium (DMEM) supplemented with $10 \%$ fetal calf serum. Kelly cells were cultured in Roswell Park Memorial Institute (RPMI)-1640 medium supplemented with $10 \%$ fetal calf serum and $1 \% \mathrm{~L}$-glutamine. All cell lines were maintained in a humidified incubator at $37^{\circ} \mathrm{C}$ and $5 \% \mathrm{CO}_{2}$ in air. The identity of all cell lines was authenticated by small tandem repeat profiling conducted at the Garvan Institute or Cellbank Australia.

siRNA transient transfection. Tumor cells were transfected with siRNAs using Lipofectamine 2000 reagent according to the manufacturer's instructions (Invitrogen, Carlsbad, CA, USA). Pre-designed control siRNA and siRNA specifically targeting NCYM or N-Myc were purchased from Qiagen (Qiagen, Hamburg, Germany), and the lncUSMycN siRNAs were custom synthesized by Dharmacon (Lafayette, CO, USA) $(14,17)$

Quantitative real-time RT-PCR. NCYM and N-Myc expression was examined by quantitative real-time RT-PCR with Power SYBR Green Master Mix (Life Technologies, Grand Island, NY, USA) as a fluorescent dye on ABI-7900 (Applied Biosystems, Grand Island, NY, USA) according to the manufacturer's instructions and as we described previously $(18,19)$. The expression of genes of interest was determined after normalisation according the expression level of the housekeeping gene $\beta$-actin in the total RNA samples.

Immunoblotting. Cells were lysed, protein was extracted and separated by gel electrophoresis. After western transfer, membranes were probed with an anti-N-Myc $(1: 2,000)$ (Santa Cruz Biotech, Santa Cruz, CA, USA) antibody followed by a horseradish peroxidase-conjugated anti-mouse $(1: 10,000)$ antibody (Santa Cruz Biotech). Protein bands were visualized with SuperSignal (Pierce, Rockford, IL, USA). Finally, the membranes were re-probed with an anti-actin antibody (1:10,000; Sigma, St. Louis, MO, USA) as loading controls.

Cell proliferation assays. Cell proliferation was examined with Alamar blue assays as we described previously (20). Briefly, cells were plated into 96-well plates and transfected with various siRNAs. Ninety-one hours later, cells were incubated with Alamar blue (Invitrogen) for five hours, and plates were read on a micro-plate reader at $570 / 595 \mathrm{~nm}$. Results were calculated according to the readings (optical density absorbance units) and expressed as percentage changes in cell numbers.

RNA immunoprecipitation assays. RNA immunoprecipitation assays were performed using Magna RIP kit from Merck Millipore according to the manufacturer's instructions (Merck Millipore, Billerica, MA, USA), with $5 \mu \mathrm{g}$ of control IgG or antiNonO antibody for immunoprecipitation and primers targeting NCYM or the negative control U1 RNA for RT-PCR (14).

Patient tumor sample analyses. NCYM, N-Myc, NonO and $\operatorname{lncUSMycN}$ expression in neuroblastoma tissues was
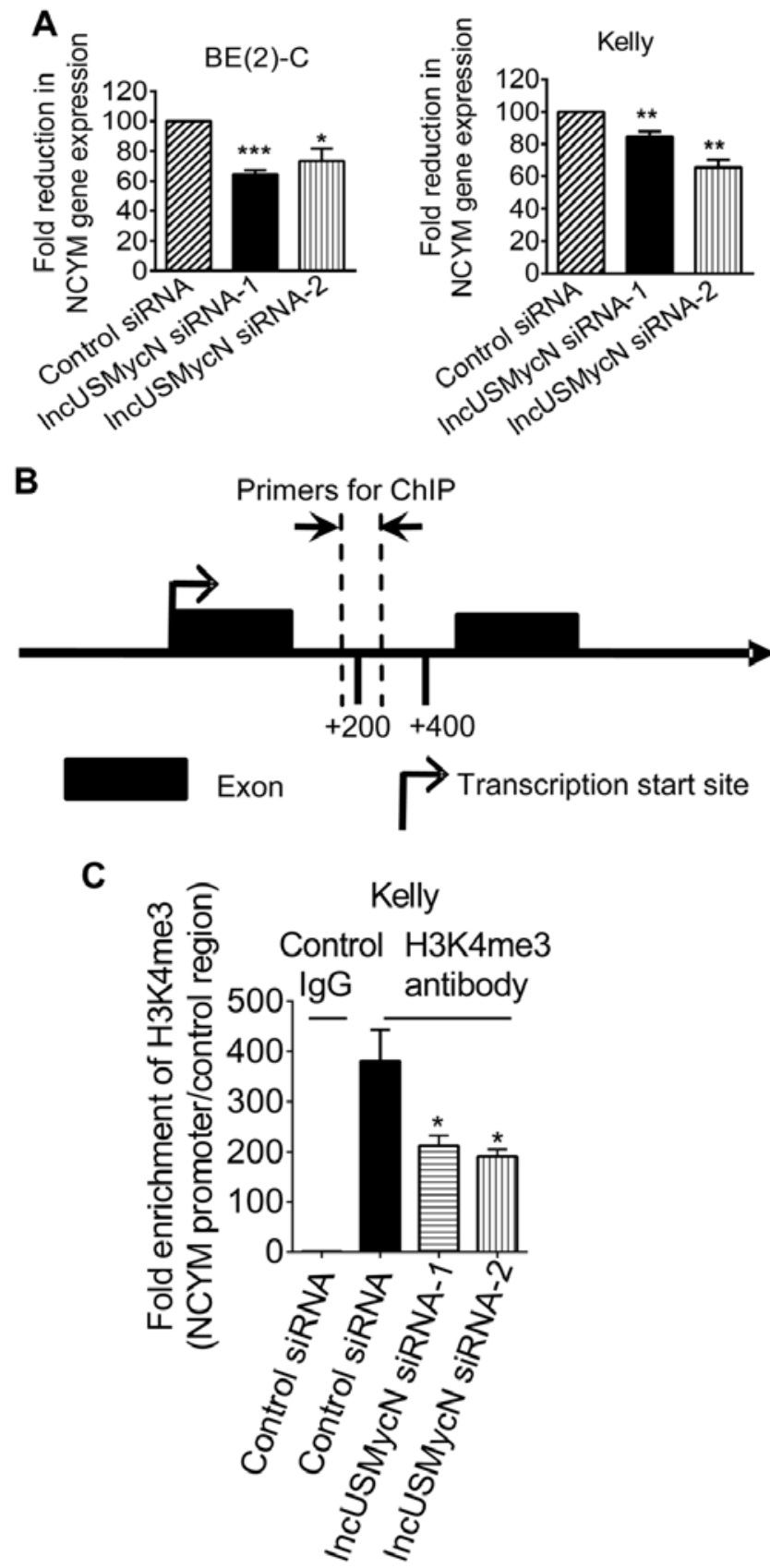

Figure 1. The long non-coding RNA 1ncUSMycN upregulates NCYM expression by inducing its gene transcription. (A) BE(2)-C and Kelly cells were transfected with scrambled control siRNA, IncUSMycN siRNA-1 or lncUSMycN siRNA-2 for $48 \mathrm{~h}$ followed by RNA extraction and RT-PCR analysis of NCYM expression. (B) Schematic representation of the NCYM gene promoter region. (C) ChIP assays were performed with a control antibody or an anti-trimethyl-H3K4 (H3K4me3) antibody and real-time PCR with primers targeting a negative control region or the intron 1 of the NCYM gene in Kelly cells after transfection with control siRNA, lncMycN siRNA-1 or lncMycN siRNA-2. Fold change in the presence of H3K4me3 at the NCYM gene promoter region was obtained after dividing fold enrichment of $\mathrm{H} 3 \mathrm{~K} 4 \mathrm{me} 3$ at the $N C Y M$ gene promoter region by fold enrichment of $\mathrm{H} 3 \mathrm{~K} 4 \mathrm{me} 3$ at the negative control region. Fold enrichment by the control antibody in cells transfected with control siRNA was arbitrarily set as 1.0 . Error bars represented standard errors. ${ }^{*} \mathrm{p}<0.05,{ }^{* *} \mathrm{p}<0.01$ and ${ }^{* * *} \mathrm{p}<0.001$.

analysed in 88 (Versteeg dataset) and 476 (Kocak dataset) human neuroblastoma samples in the publicly available gene expression databases (http://r2.amc.nl). Clinical information for the 88 patients in the Versteeg dataset was directly 

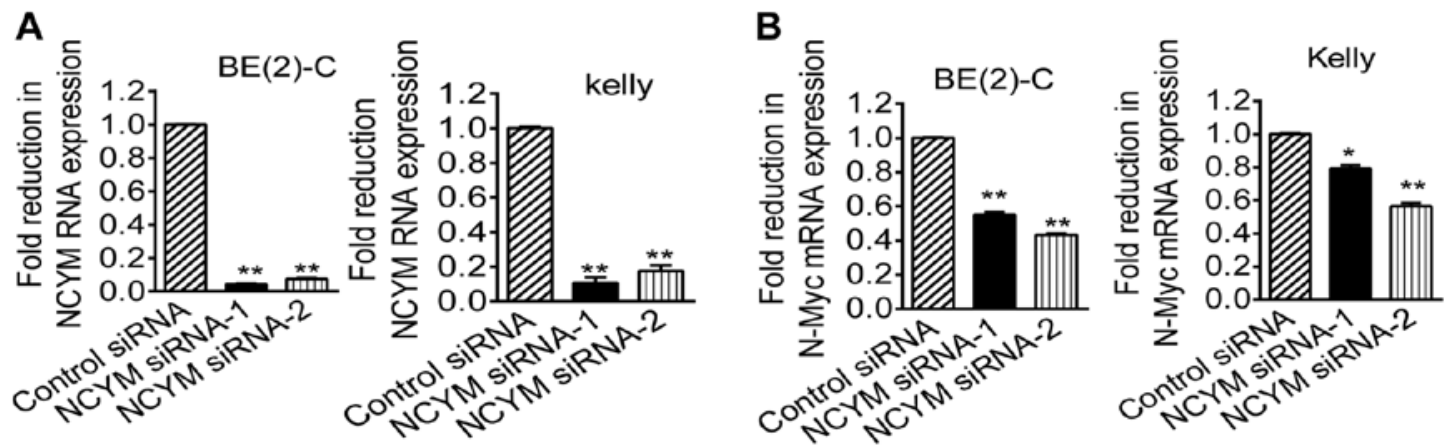

C
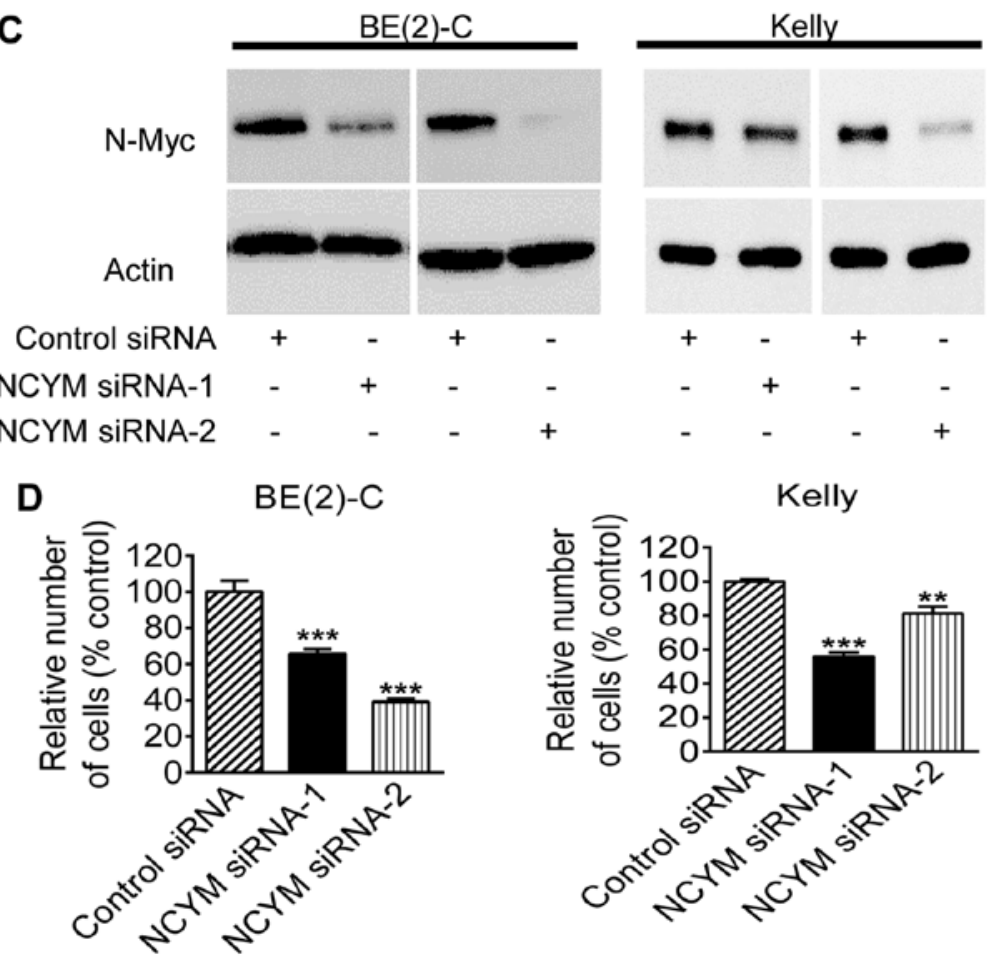

Figure 2. NCYM upregulates N-Myc expression and induces neuroblastoma cell proliferation. (A-C) BE(2)-C and Kelly cells were transfected with scrambled control siRNA, NCYM siRNA-1 or NCYM siRNA-2 for $48 \mathrm{~h}$ followed by RNA and protein extraction, real-time RT-PCR analysis of NCYM (A) and N-Myc (B). RNA expression and immunoblot analysis of N-Myc protein expression (C). (D) BE(2)-C and Kelly cells were transfected with control siRNA, NCYM siRNA-1 or NCYM siRNA-2 for $72 \mathrm{~h}$. Cell numbers were examined by Alamar blue assays and expressed as percentage changes relative to control siRNA-transfected samples. Error bars represent standard errors. ${ }^{*} \mathrm{p}<0.05,{ }^{* *} \mathrm{p}<0.01$ and ${ }^{* * *} \mathrm{p}<0.001$.

downloaded from http://r2.amc.nl, and clinical information for the 476 patients in the Kocak dataset was extracted from the authors' reports $(21,22)$.

Correlation between NCYM expression and N-Myc, NonO and lncUSMycN expression in human neuroblastoma tissues was examined with Pearson's correlation. For patient prognosis studies, overall survival was defined as the time from diagnosis until death, or otherwise as the time until last contact. The patient cohort was dichotomized into two groups (low versus high NCYM expression). Survival analyses were performed according to the method of Kaplan and Meier and comparisons of survival curves were made using two-sided log-rank tests (23).

Statistical analyses. All data for statistical analysis were calculated as mean \pm standard error. Differences were analyzed for significance using two-sided unpaired t-test for two groups, or multiple comparison one-way analysis of variance (ANOVA) for more than two groups. A probability value of $\leq 0.05$ was considered significant.

\section{Results}

The long non-coding RNA lncUSMycN upregulates NCYM expression by inducing its gene transcription. The lncUSMycN, NCYM and MYCN genes are within the $130 \mathrm{~kb}$ core genomic DNA region amplified in a quarter of human neuroblastoma tissues (3). As long non-coding RNAs are well-known to upregulate the expression of neighboring genes in cis (24), we investigated whether IncUSMycN modulated NCYM gene expression. BE(2)-C and Kelly neuroblastoma cells were transfected with control siRNA or two independent siRNAs targeting different regions of lncUSMycN. The lncUSMycN siRNAs had been previously validated to knock down lncUSMycN RNA expression in BE(2)-C and Kelly cells (14). As shown in Fig. 1A, transfection with lncUSMycN siRNAs 


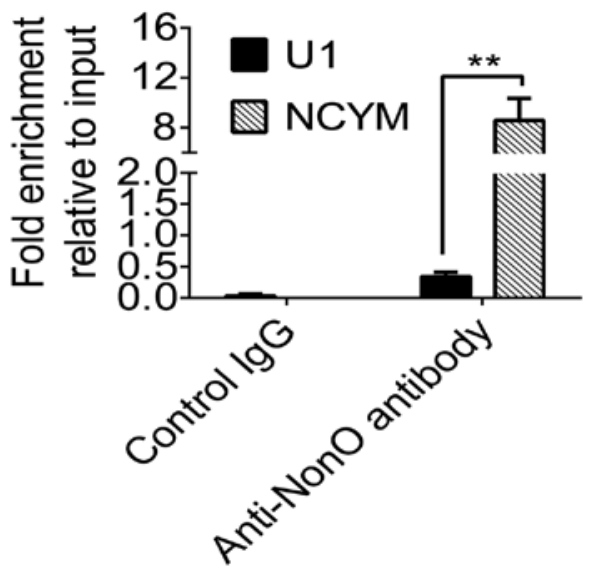

Figure 3. NCYM RNA binds to the RNA-binding protein NonO. RNA immunoprecipitation assays were performed with a control or an anti-NonO antibody in Kelly cells. RNA samples immunoprecipitated by the control IgG or the anti-NonO antibody were subjected to RT-PCR analysis of NCYM RNA or the negative control U1 RNA. Error bars represent standard errors. *** $\mathrm{p}<0.01$.

significantly reduced NCYM RNA expression in the $M Y C N$ oncogene-amplified BE(2)-C and Kelly neuroblastoma cells.

To understand whether lncUSMycN upregulated NCYM expression through activating NCYM gene transcription, we performed chromatin immunoprecipitation (ChIP) assays with a control antibody $(\mathrm{Ab})$ or an antibody against trimethylated histone H3 lysine 4 (H3K4me3), a marker for active gene transcription. As shown in Fig. 1B and C, the ChIP assays showed that knocking down lncUSMycN expression with siRNAs reduced histone H3K4 trimethylation at the NCYM gene promoter (intron 1). Taken together, the data suggest that lncUSMycN upregulates $N C Y M$ expression by enhancing its gene transcription.

NCYM upregulates $N$-Myc expression and induces neuroblastoma cell proliferation. As the NCYM gene is antisense to the $M Y C N$ oncogene, we examined whether NCYM regulated $\mathrm{N}-\mathrm{Myc}$ expression. BE(2)-C and Kelly cells were transfected with two independent siRNAs targeting different regions of NYCM RNA or a control siRNA for $48 \mathrm{~h}$. As shown in Fig. 2A, transfection with NCYM siRNAs efficiently reduced NCYM expression. RT-PCR analysis showed that knocking down NCYM expression with siRNAs reduced N-Myc mRNA expression (Fig. 2B), and immunoblot analysis showed that knocking down NCYM expression also reduced N-Myc protein expression (Fig. 2C). Consistent with the RT-PCR data, the reduction in N-Myc protein expression by NCYM siRNA-1 was weaker than NCYM siRNA-2, indicating variation in the efficacy of NCYM siRNA-1 and NCYM siRNA-2.

As N-Myc is well-known to induce neuroblastoma cell proliferation, we performed Alamar blue cell proliferation assays in $\mathrm{BE}(2)-\mathrm{C}$ and Kelly cells after transfection with control siRNA, NCYM siRNA-1 or NCYM siRNA-2 for $48 \mathrm{~h}$. Alamar blue assays showed that knocking down NCYM expression with siRNAs reduced the number of neuroblastoma cells (Fig. 2D). Taken together, these data suggest that NCYM
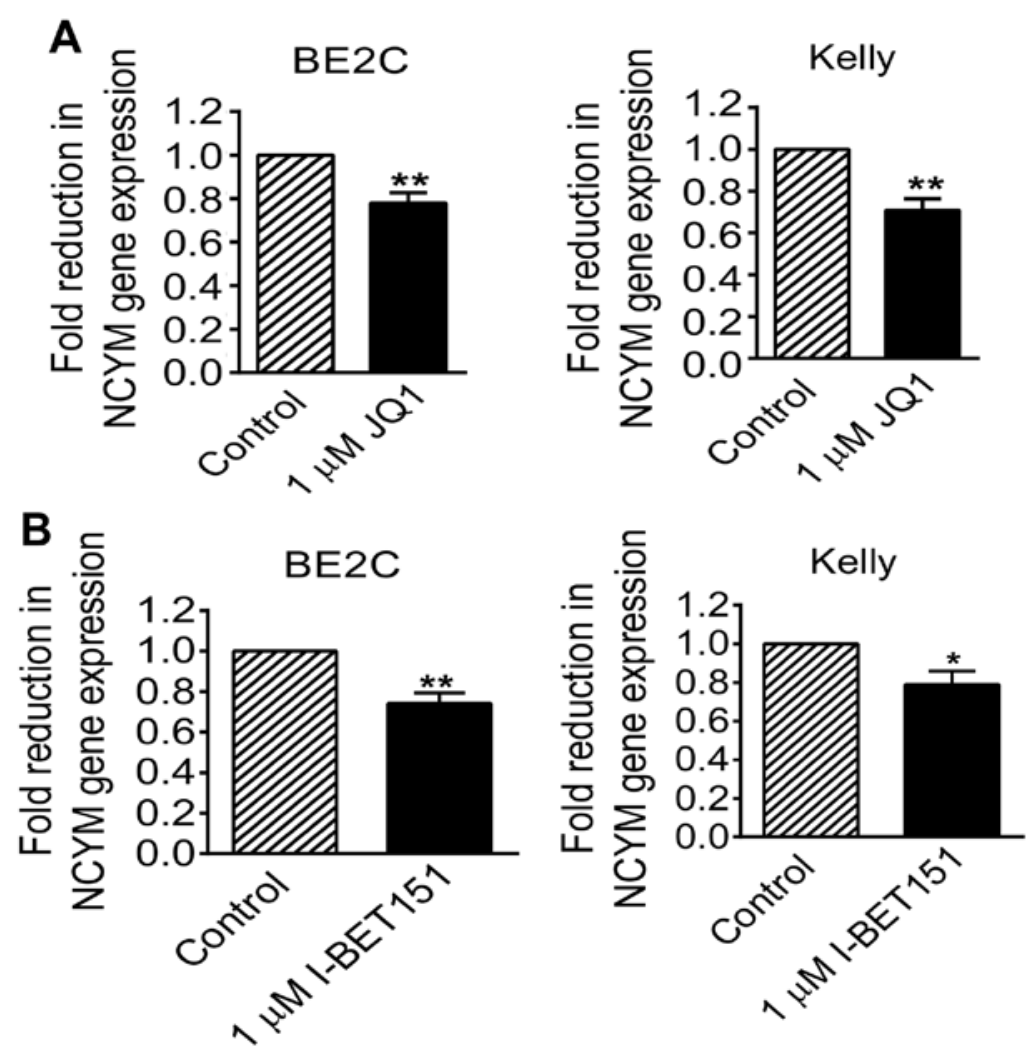

Figure 4. BET bromodomain inhibitors reduce NCYM gene expression. (A) BE(2)-C and Kelly cells were treated with vehicle control or $1 \mu \mathrm{M} \mathrm{JQ1} \mathrm{for} 48 \mathrm{~h}$, followed by RNA extraction and real-time RT-PCR analysis of NCYM expression. (B) BE(2)-C and Kelly cells were treated with vehicle control or $1 \mu \mathrm{M}$ I-BET151 for $48 \mathrm{~h}$ followed by RNA extraction and real-time RT-PCR analysis of NCYM expression. 
A

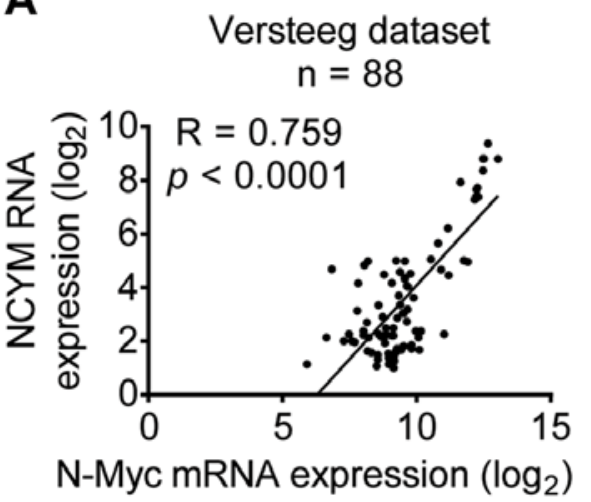

B

Versteeg dataset

$$
\mathrm{n}=88
$$

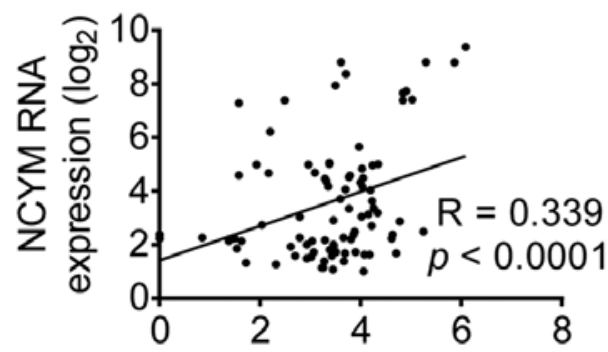

IncUSMycN RNA expression $\left(\log _{2}\right)$

C

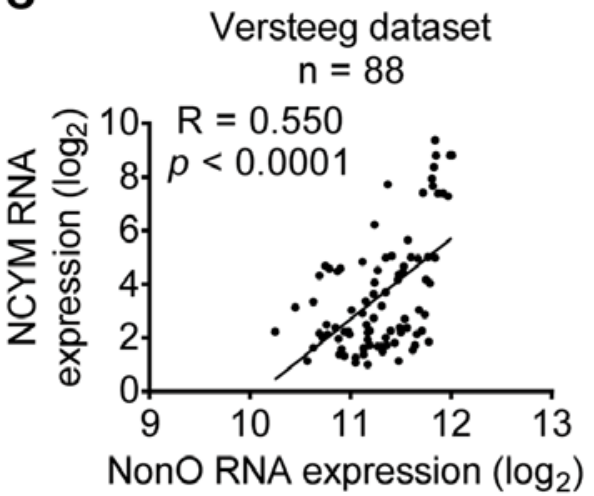

D

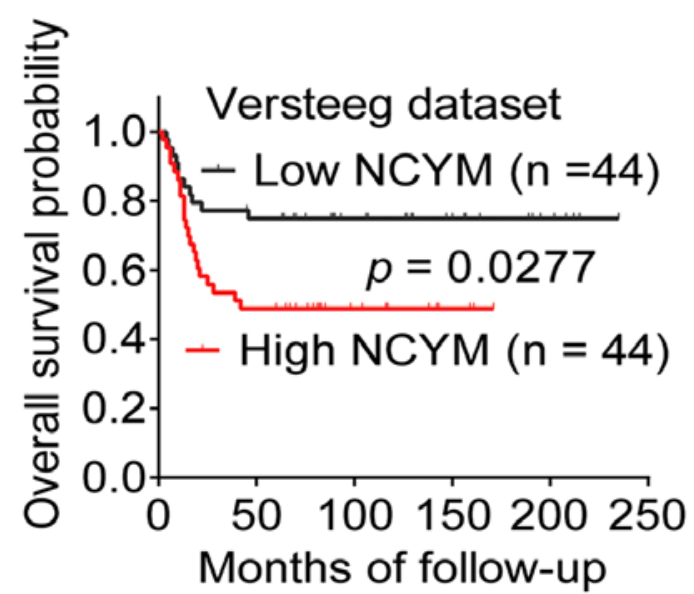

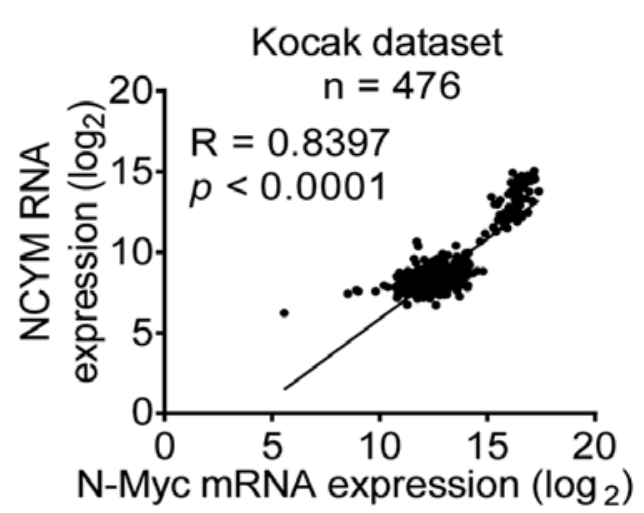
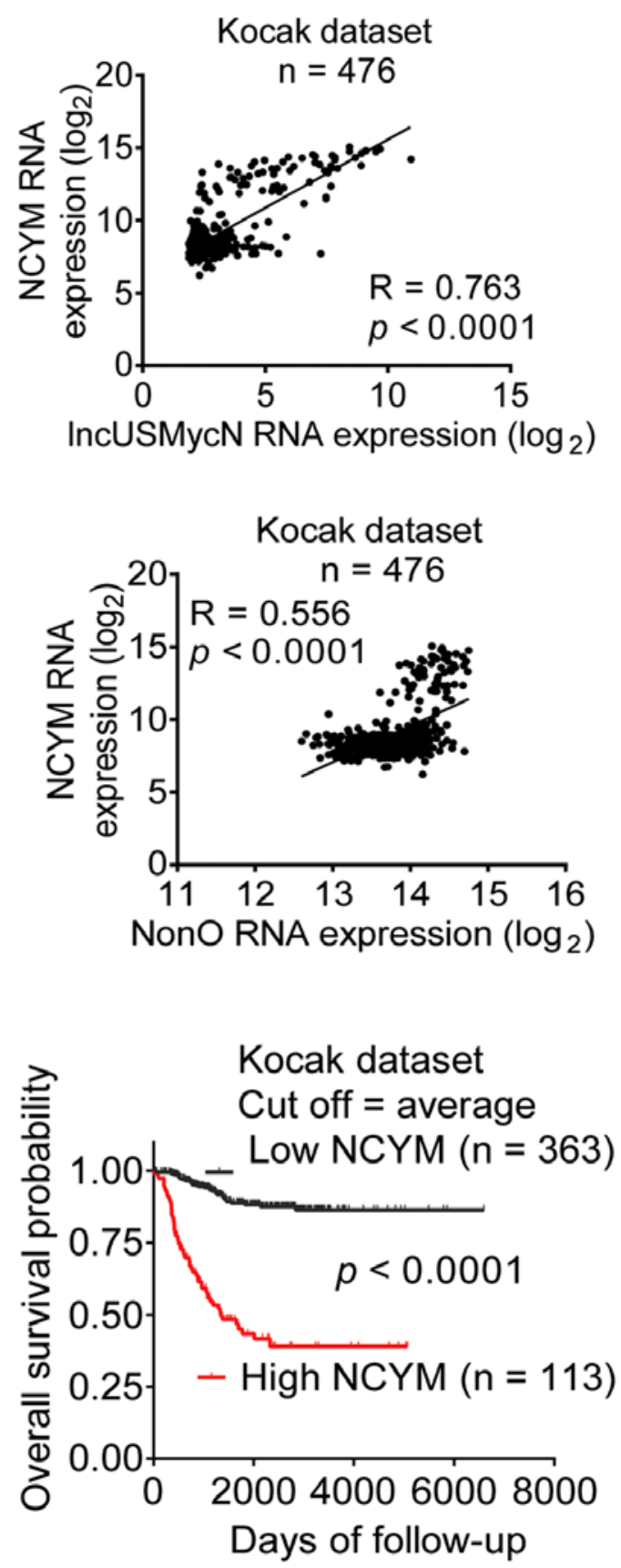

Figure 5. NCYM expression in neuroblastoma tissues positively correlates with N-Myc, NonO and lncUSMycN expression and poor patient prognoses. (A-C) Two-sided Pearson's correlation was used to analyze correlation between NCYM expression and N-Myc (A), NonO (B) and lncUSMycN (C) expression in tumor tissues from 88 and 476 neuroblastoma patients in the publicly available microarray gene expression Versteeg dataset and Kocak dataset, respectively. (D) Kaplan-Meier curves showed the probability of overall survival of patients according to the levels of NCYM expression in the 88 and 476 neuroblastoma patients in the Versteeg and Kocak datasets, respectively. 
upregulates N-Myc expression, leading to neuroblastoma cell proliferation.

NCYM RNA binds to the RNA-binding protein NonO. We have previously shown that the RNA-binding protein NonO upregulates N-Myc mRNA expression (14). Next we performed RNA immunoprecipitation assays with an anti-NonO antibody or control IgG, followed by RT-PCR analysis of NCYM RNA. As shown in Fig. 3, the anti-NonO antibody, compared with the control IgG, efficiently immunoprecipitated NCYM RNA, compared with the negative control U1 RNA. The data suggest that NCYM RNA forms a complex with NonO protein, leading to N-Myc mRNA upregulation.

BET bromodomain inhibitors reduce NCYM gene expression. The BET bromodomain inhibitors JQ1 and I-BET151 have been shown to reduce oncogene expression $(25,26)$. We next examined whether the BET bromodomain inhibitors modulated NCYM expression. BE(2)-C and Kelly cells were treated with vehicle control or the BET bromodomain inhibitor JQ1 or I-BET151. RT-PCR analysis showed that treatment with JQ1 (Fig. 4A) or I-BET151 (Fig. 4B) reduced NCYM RNA expression in both $\mathrm{BE}(2)-\mathrm{C}$ and Kelly cells. The data suggest that NCYM gene expression is regulated by BET bromodomain proteins, and that BET bromodomain inhibitors can be employed to suppress NCYM expression.

NCYM expression in neuroblastoma tissues positively correlates with $\mathrm{N}-\mathrm{Myc}$, NonO and IncUSMycN expression and poor patient prognoses. We finally examined correlation of NCYM expression in human neuroblastoma tissues with $\mathrm{N}-\mathrm{Myc}$, NonO and lncUSMycN expression and patient prognosis, using the publicly available (http://r2.amc.nl) Versteeg and Kocak $(21,22)$ microarray gene expression datasets. Probes for NCYM, N-Myc, NonO and lncUSMycN in the Affymetrix HG-U133_Plus_2 microarray in the Versteeg dataset were 207028_at, 209757_s_at, 200057_s_at and 244438_at, respectively. Probes for NCYM, N-Myc, NonO and lncUSMycN in the Agilent Technologies oligonucleotide microarrays in the Kocak dataset were UKv4_A_23_P67952, UKv4_A_24_ P94402, UKv4_A_24_P151727 and UKv4_A_24_P486173, respectively. As shown in Fig. 5A-C, NCYM RNA expression was positively correlated with N-Myc, NonO and lncUS$\mathrm{MycN}$ in the cohorts of 88 and 476 neuroblastoma patients, respectively. Kaplan-Meier analysis showed that high levels of NCYM expression in neuroblastoma tissues correlated with poor patient prognoses (Fig. 5D). Taken together, the data suggest that high levels of NCYM expression in human neuroblastoma tissues positively correlate with $\mathrm{N}-\mathrm{Myc}$, NonO and lncUSMycN expression and predict poor patient survival.

\section{Discussion}

The $M Y C N$ antisense gene $N C Y M$, like the MYCN and the long non-coding RNA lncUSMYCN genes, is amplified in approximately a quarter of human neuroblastoma tissues (15). Long non-coding RNAs are now well-known to exert biological effects through both transcriptional and posttranscriptional mechanisms and to regulate gene transcription both in cis and in trans $(24,27)$. We have recently reported that the long non-coding RNA lncUSMycN upregulates N-Myc mRNA expression by binding to the RNA-binding protein NonO, but does not directly regulate $M Y C N$ gene transcription since knocking down lncUSMycN expression does not have an effect on histone H3K4 trimethylation, a marker for active gene transcription, at the $M Y C N$ gene promoter (14). In this study, we found that IncUSMycN upregulates NCYM expression in human neuroblastoma cells, and that knocking down lncUSMycN expression reduces histone $\mathrm{H} 3 \mathrm{~K} 4$ trimethylation at the NCYM gene promoter. The data suggest that lncUS$\mathrm{MycN}$ upregulates NCYM expression by activating its gene transcription.

This study demonstrates that NCYM upregulates N-Myc mRNA and protein expression, and that NCYM RNA binds to the RNA-binding protein NonO. As our previous study shows that NonO protein binds to N-Myc mRNA and posttranscriptionally upregulates N-Myc mRNA expression (14), we propose that NCYM RNA upregulates N-Myc mRNA expression by binding to NonO protein. In addition, we have confirmed that knocking down NCYM expression leads to neuroblastoma cell growth inhibition. Taken together, the data suggest that NCYM RNA overexpression upregulates N-Myc mRNA expression by binding to NonO protein, leading to neuroblastoma cell proliferation.

This study shows that high levels of NCYM expression in two independent cohorts of human neuroblastoma tissues positively correlate with high levels of N-Myc, NonO and lncUSMycN expression. Importantly, Kaplan-Meier analysis shows that high levels of NCYM expression in neuroblastoma tissues correlate with poor prognosis in the two cohorts of 88 and 476 patients. These data therefore demonstrate that high levels of NCYM expression in human neuroblastoma tissues can be employed as a marker for poor prognosis and a therapeutic target.

Histone lysine acetylation plays a pivotal role in regulating chromatin dynamics and gene transcription. The BET bromodomain is a conserved structural unit in chromatin-associated proteins and histone acetyltranferases, known to recognize acetyl-lysine residues on proteins (28). BET bromodomain inhibitors have been shown to exert anticancer effects against neuroblastoma by blocking BET bromodomain protein binding to the MYCN gene intron 1, a region shared by the $M Y C N$ and the NCYM genes, and consequently reducing N-Myc expression $(17,26)$. In this study, we found that suppression of BET bromodomain proteins by the BET bromodomain inhibitors JQ1 and I-BET151 considerably reduces NCYM expression, and that knocking down NCYM expression with siRNAs considerably reduces neuroblastoma cell proliferation. Our data suggest that BET bromodomain inhibitors reduce $\mathrm{N}-\mathrm{Myc}$ expression directly by repressing $M Y C N$ gene transcription and indirectly by repressing $N C Y M$ gene transcription, and exert anticancer effects against neuroblastoma by simultaneously blocking the expression of N-Myc and NCYM.

In conclusion, $N C Y M, M Y C N$ and $\operatorname{lnc} U S M y c N$ genes are co-amplified in human neuroblastoma. Our data demonstrate that the long non-coding RNA IncUSMycN upregulates NCYM expression by activating its gene transcription, and that NCYM RNA upregulates N-Myc expression by binding to the RNA-binding protein NonO. High levels of NCYM expression in human neuroblastoma tissues correlate with 
high levels of N-Myc, NonO and lncUSMycN expression and poor patient survival, and treatment with BET bromodomain inhibitors reduces NCYM expression. Our findings therefore provide further evidence for the application of BET bromodomain inhibitors for the therapy of neuroblastoma characterized by $M Y C N / N C Y M$ gene locus amplification.

\section{Acknowledgements}

This study was supported by National Health and Medical Research Council (NHMRC) Australia grants. T.L. is a recipient of an Australian Research Council Future Fellowship. Children's Cancer Institute Australia is affiliated with UNSW Australia and Sydney Children's Hospital.

\section{References}

1. Colon NC and Chung DH: Neuroblastoma. Adv Pediatr 58: 297-311, 2011.

2. Matthay KK, George RE and Yu AL: Promising therapeutic targets in neuroblastoma. Clin Cancer Res 18: 2740-2753, 2012.

3. Reiter JL and Brodeur GM: High-resolution mapping of a $130-\mathrm{kb}$ core region of the MYCN amplicon in neuroblastomas. Genomics 32: 97-103, 1996.

4. Maris JM, Hogarty MD, Bagatell R and Cohn SL: Neuroblastoma. Lancet 369: 2106-2120, 2007.

5. Matthay KK, Villablanca JG, Seeger RC, Stram DO, Harris RE, Ramsay NK, Swift P, Shimada H, Black CT, Brodeur GM, et al; Children's Cancer Group: Treatment of high-risk neuroblastoma with intensive chemotherapy, radiotherapy, autologous bone marrow transplantation, and 13-cis-retinoic acid. N Engl J Med 341: 1165-1173, 1999.

6. Knoepfler PS, Cheng PF and Eisenman RN: N-myc is essential during neurogenesis for the rapid expansion of progenitor cell populations and the inhibition of neuronal differentiation. Genes Dev 16: 2699-2712, 2002.

7. Huang $M$ and Weiss WA: Neuroblastoma and MYCN. Cold Spring Harb Perspect Med 3: a014415, 2013.

8. Mercer TR, Dinger ME and Mattick JS: Long non-coding RNAs: Insights into functions. Nat Rev Genet 10: 155-159, 2009.

9. Ponting CP, Oliver PL and Reik W: Evolution and functions of long noncoding RNAs. Cell 136: 629-641, 2009.

10. Batista PJ and Chang HY: Long noncoding RNAs: Cellular address codes in development and disease. Cell 152: 1298-1307, 2013.

11. Huarte M: The emerging role of lncRNAs in cancer. Nat Med 21: $1253-1261,2015$.

12. Huarte M, Guttman M, Feldser D, Garber M, Koziol MJ, Kenzelmann-Broz D, Khalil AM,Zuk O, Amit I, Rabani M, et al: A large intergenic noncoding RNA induced by p53 mediates global gene repression in the p53 response. Cell 142: 409-419, 2010.

13. Lee S, Kopp F, Chang TC, Sataluri A, Chen B, Sivakumar S, Yu H, Xie Y and Mendell JT: Noncoding RNA NORAD regulates genomic stability by sequestering PUMILIO proteins. Cell 164: 69-80, 2016.

14. Liu PY, Erriquez D, Marshall GM, Tee AE, Polly P, Wong M, Liu B, Bell JL, Zhang XD, Milazzo G, et al: Effects of a novel long noncoding RNA, lncUSMycN, on N-Myc expression and neuroblastoma progression. J Natl Cancer Inst 106: dju113, 2014.
15. Suenaga Y, Islam SM, Alagu J, Kaneko Y, Kato M, Tanaka Y, Kawana $\mathrm{H}$, Hossain S, Matsumoto D, Yamamoto M, et al: NCYM, a Cis-antisense gene of MYCN, encodes a de novo evolved protein that inhibits GSK3 $\beta$ resulting in the stabilization of MYCN in human neuroblastomas. PLoS Genet 10: e1003996, 2014.

16. Shoji W, Suenaga Y, Kaneko Y, Islam SM, Alagu J, Yokoi S, Nio M and Nakagawara A: NCYM promotes calpain-mediated Myc-nick production in human MYCN-amplified neuroblastoma cells. Biochem Biophys Res Commun 461: 501-506, 2015.

17. Shahbazi J, Liu PY, Atmadibrata B, Bradner JE, Marshall GM, Lock RB and Liu T: The bromodomain inhibitor JQ1 and the histone deacetylase inhibitor panobinostat synergistically reduce $\mathrm{N}-\mathrm{Myc}$ expression and induce anticancer effects. Clin Cancer Res 22: 2534-2544, 2016.

18. Liu PY, Xu N, Malyukova A, Scarlett CJ, Sun YT, Zhang XD, Ling D, Su SP, Nelson C, Chang DK, et al: The histone deacetylase SIRT2 stabilizes Myc oncoproteins. Cell Death Differ 20: 503-514, 2013.

19. Tee AE, Liu B, Song R, Li J, Pasquier E, Cheung BB, Jiang C, Marshall GM, Haber M, Norris MD, et al: The long noncoding RNA MALAT1 promotes tumor-driven angiogenesis by up-regulating pro-angiogenic gene expression. Oncotarget 7: 8663-8675, 2016

20. Liu T, Liu PY, Tee AEL, Haber M, Norris MD, Gleave ME and Marshall GM: Over-expression of clusterin is a resistance factor to the anti-cancer effect of histone deacetylase inhibitors. Eur J Cancer 45: 1846-1854, 2009.

21. Kocak H, Ackermann S, Hero B, Kahlert Y, Oberthuer A, Juraeva D, Roels F, Theissen J, Westermann F, Deubzer H, et al: Hox-C9 activates the intrinsic pathway of apoptosis and is associated with spontaneous regression in neuroblastoma. Cell Death Dis 4: e586, 2013.

22. Oberthuer A, Juraeva D, Li L, Kahlert Y, Westermann F, Eils R, Berthold F, Shi L, Wolfinger RD, Fischer M, et al: Comparison of performance of one-color and two-color gene-expression analyses in predicting clinical endpoints of neuroblastoma patients. Pharmacogenomics J 10: 258-266, 2010.

23. Kaplan EL and Meier P: Nonparametric estimation from incomplete observations. In: Breakthroughs in Statistics. Kotz S and Johnson N (eds)., Springer, New York, NY, pp319-337, 1992.

24. Ørom UA, Derrien T, Beringer M, Gumireddy K, Gardini A, Bussotti G, Lai F, Zytnicki M, Notredame C, Huang Q, et al: Long noncoding RNAs with enhancer-like function in human cells. Cell 143: 46-58, 2010.

25. Wyce A, Ganji G, Smitheman KN, Chung CW, Korenchuk S, Bai Y, Barbash O, Le B, Craggs PD, McCabe MT, et al: BET inhibition silences expression of MYCN and BCL2 and induces cytotoxicity in neuroblastoma tumor models. PLoS One 8: e72967, 2013.

26. Puissant A, Frumm SM, Alexe G, Bassil CF, Qi J, Chanthery YH, Nekritz EA, Zeid R, Gustafson WC, Greninger P, et al: Targeting MYCN in neuroblastoma by BET bromodomain inhibition. Cancer Discov 3: 308-323, 2013.

27. Ulitsky I and Bartel DP: lincRNAs: Genomics, evolution, and mechanisms. Cell 154: 26-46, 2013.

28. Sanchez R and Zhou MM: The role of human bromodomains in chromatin biology and gene transcription. Curr Opin Drug Discov Devel 12: 659-665, 2009. 